\title{
Doppelte ethische Beurteilung von im Ausland durchgeführten Studien, insbesondere von Nord-Süd-Forschungspartnerschaften
}

\section{Arbeitsgruppe «Koordination der Beurteilung klinischer Versuche» (AG KoBeK)*}

\footnotetext{
* Die AG KoBeK umfasst Vertreter/ innen von Bundesbehörden (Swissmedic und Bundesamt für Gesundheit), kantonalen Behörden (GDK und Kantonsapotheker) sowie der Ethikkommissionen (Arbeitsgemeinschaft der Schweizer Ethikkommissionen, AGEK) und steht unter dem Patronat der SAMW. Sie wird präsidiert von Prof. Dr. iur. Dominique Sprumont (als Vertreter der SAMW). Die AG KoBeK bietet eine Plattform für den Informations- und Erfahrungsaustausch der im Bereich Überwachung und Kontrolle von klinischen Versuchen aktiven Partner. Auf Bitte der beteiligten Institutionen kann sie Interpretationshilfen für ethische und gesetzliche Vorschriften bereitstellen.
}

Wenn sie den wachsenden Bedürfnissen des Gesundheitssektors Rechnung tragen will, kann die Forschung nicht an den staatlichen Grenzen haltmachen. Auch die Globalisierung beeinflusst das Gesundheitswesen. Folglich entsteht die Tendenz, Forschungsaktivitäten vermehrt international einzubetten. Darüber hinaus erfordern die beträchtlichen Bedürfnisse des Gesundheitssektors in den Entwicklungsländern, dass die Forschungsbemühungen in Nord-Süd-Partnerschaften erheblich gesteigert werden. Bisher wurden den spezifischen Krankheitsbildern dieser Länder nur gerade 10\% der gesamten internationalen Gesundheitsforschung gewidmet. Dies trotz der Tatsache, dass dort 90\% der Weltbevölkerung lebt. Das vorliegende Dokument definiert Nord-Süd-Partnerschaften als Forschungsgemeinschaften, die die Bemühungen und den Kräfteeinsatz von Nord wie Süd auf ein Ziel hin koordinieren, das von beiden Seiten anerkannt wird.

In diesem Zusammenhang können der Schutz von Versuchspersonen und die Qualität der Forschungsresultate nur garantiert werden, wenn die in der Forschung anerkannten ethischen Prinzipien regelmässig zur Anwendung kommen, auch bei der Konfrontation mit neuen Forschungssituationen. Deshalb ist es wichtig, dass alle Beteiligten ihre Rechte und Pflichten kennen und sich der Grenzen ihrer Tätigkeit bewusst werden. Dies gilt insbesondere für die Ethikkommissionen für Forschungsuntersuchungen (EKF).

\section{Zweck und Geltungsbereich}

Die nachstehenden Vorschläge verstehen sich als Orientierungshilfe für den Fall, dass einer EKF ein Forschungsprojekt, das im Ausland durchgeführt werden soll, zur Beurteilung unterbreitet wird. Sie zeigen auf, wann und unter welchen Bedingungen eine doppelte ethische Beurteilung notwendig wird. Eine solche ist insbesondere bei Forschungsvorhaben angezeigt, die teil- weise oder vollständig im Ausland durchgeführt werden, während die Sponsoren oder die Forschenden ihre Aktivitäten von der Schweiz aus lenken. In diesem Zusammenhang muss geklärt werden, in welchem Umfang die in der Schweiz zuständige EKF das Forschungsprojekt zu beurteilen hat, dies im Vergleich zur EKF, die am Durchführungsort zuständig ist (siehe 4).

Die nachstehenden Vorschläge kommen bei Forschungsuntersuchungen zur Anwendung, die im Rahmen von sogenannten Nord-SüdPartnerschaften durchgeführt werden. In Anlehnung an die CIOMS-Richtlinien (Council for International Organizations of Medical Sciences) handelt es sich dabei um Forschungsuntersuchungen, die in einem Entwicklungsland stattfinden, aber von einem externen Unternehmen oder einer externen Organisation mit Sitz in einem nördlichen Industrieland gefördert, finanziert und manchmal vollständig oder teilweise durchgeführt werden. Umgekehrt können Forschungen von einem Entwicklungsland finanziert werden, während sich Forschende aus einem nördlichen Industrieland an den Untersuchungen beteiligen.

Die Richtlinien können auch für Studien in Europa, den USA oder Japan angewendet werden, falls der Schutz der Versuchspersonen es erfordert. Dies ist zum Beispiel der Fall, wenn Versuchspersonen in einem Land rekrutiert werden, die Studie aber in einem anderen Land stattfindet. In diesem Fall bedürfen die Betreuung und der Schutz der Versuchspersonen besonderer Aufmerksamkeit.

Solche Projekte unterscheiden sich von multizentrischen Studien, die auf der Grundlage eines einheitlichen Studienprotokolls in verschiedenen Forschungszentren und folglich von verschiedenen Prüfärzten durchgeführt werden. Wenn eine multizentrische Studie in verschiedenen Ländern durchgeführt wird, so bedarf deren Durchführung der positiven Beurteilung der zuständigen EKF jedes betroffenen Landes. 


\section{Quellen}

Auf internationaler Ebene wurde der Grundsatz der doppelten ethischen Beurteilung erstmals in den CIOMS-Richtlinien von 1982 verwendet, und er wurde durch deren Revisionen 1993 und 2003 bestätigt. Auch die Deklaration von Helsinki erinnert daran, dass Forschende sich «der in ihren eigenen Ländern sowie der auf internationaler Ebene für die Forschung am Menschen geltenden ethischen, gesetzlichen und verwaltungstechnischen Vorschriften bewusst sein» sollten, während die zuständige EKF «die Gesetze und Bestimmungen des Landes, in dem das Forschungsvorhaben durchgeführt wird, beachten» muss. Falls die angestammte Tätigkeit der Forschenden und der Durchführungsort des Forschungsvorhabens in zwei verschiedenen Ländern anzusiedeln sind, muss sichergestellt sein, dass die Vorschriften beider Länder respektiert werden. In der Schweiz verweist die Standesordnung der FMH bezüglich der Forschungsuntersuchungen am Menschen explizit auf die Deklaration von Helsinki. Die Einhaltung dieser Vorschriften gehört folglich zu den generellen Berufspflichten der schweizerischen Ärzteschaft. Darüber hinaus ist eine doppelte ethische Beurteilung Voraussetzung, damit Forschungsresultate aus Nord-Süd-Partnerschaften in den wichtigsten internationalen Wissenschaftszeitschriften publiziert werden. Schliesslich sieht das Zusatzprotokoll zum Übereinkommen über Menschenrechte und Biomedizin des Europarates (2005) vor: «Jedes Forschungsvorhaben ist zur unabhängigen Prüfung seiner ethischen Vertretbarkeit einer Ethikkommission vorzulegen. Solche Vorhaben sind in jedem Land, in dem eine Forschungstätigkeit stattfinden soll, zur unabhängigen Prüfung vorzulegen» (unsere Hervorhebung).

Im schweizerischen Recht sind diese Texte nicht direkt anwendbar. Jedoch sieht das Heilmittelgesetz vor, dass die internationalen Richtlinien der guten Praxis der klinischen Versuche (ICH GCP) zu respektieren sind (Art. 53 HMG). Der Bundesrat hat in der Verordnung über die klinischen Versuche (VKlin) entsprechend präzisiert, dass die klinischen Versuche mit Heilmitteln ICH-GCP-konform zu sein haben. Da sich die ICH GCP explizit auf die Deklaration von Helsinki beziehen, ist diese folglich im Bereich der klinischen Versuche mit Heilmitteln anwendbar. Darüber hinaus hält die Verordnung über die Zulassung von Arzneimitteln (AMZV) fest: «Die Dokumentation über die klinischen Prüfungen muss insbesondere belegen, a. dass die Untersuchungen am Menschen nach den aner- kannten Regeln der Guten Praxis der klinischen Versuche durchgeführt worden sind» (unsere Hervorhebung). Selbst wenn also die Schweizer Behörden auf direktem Weg keine doppelte ethische Beurteilung einfordern können, bleibt die Beurteilung durch eine schweizerische EKF für die Sponsoren eine geeignete Massnahme, um sicherzustellen, dass ein Projekt mit den ICH GCP und der Deklaration von Helsinki in Einklang steht. Schliesslich stellt die Betreuung und Überprüfung der Studienprotokolle durch eine EKF in der Schweiz ein erstes Element des externen und unabhängigen Monitorings dar, das für Forschungen in Entwicklungsländern besonders wichtig ist, um den Schutz der Versuchspersonen zu garantieren.

Zusammenfassend ist es wichtig, dass die EKF ihre Verantwortung wahrnehmen, um a) den Gesundheitsbedürfnissen der Entwicklungsländer gerecht zu werden und b) den Schweizer Forschenden sowie den öffentlichen bzw. privaten Forschungsinstitutionen zu ermöglichen, ihre Nord-Süd-Partnerschaften weiterzuführen.

\section{Grundsätze der Beurteilung}

\subsection{Forschungsvorhaben werden einer EKF unterbreitet}

Ein Forschungsprojekt mit Menschen darf erst begonnen werden, wenn an jedem Ort, an dem das Forschungsvorhaben realisiert wird, eine positive Stellungnahme der zuständigen und anerkannten EKF vorliegt. Im Falle einer NordSüd-Partnerschaft bedarf es mindestens einer Beurteilung in einem nördlichen Land sowie einer Beurteilung in jedem anderen Land, in dem die Forschung durchgeführt wird. Aufgrund des Respekts staatlicher Unabhängigkeit ist es aber durchaus möglich, dass es mehrere EKF-Beurteilungen pro Land gibt.

Der Grundsatz der doppelten Beurteilung stützt sich auf die Anerkennung und die Respektierung der ethischen und juristischen Regelwerke, die in den betreffenden Ländern Geltung haben. Darüber hinaus trägt er der Tatsache Rechnung, dass es zwischen den beteiligten Ländern erhebliche kulturelle Differenzen geben kann, aber auch kleinere Gemeinschaften möglicherweise ihre ganz eigenen Wertesysteme kennen. Diese Unterschiede müssen sich folglich in den zur Beurteilung beigezogenen EKF spiegeln. Falls in den betreffenden Ländern die Wertvorstellungen ähnlich sind (wenn z. B. die Sponsoren und der Hauptprüfer in zwei nördlichen Ländern ansässig sind), so kann auf die doppelte ethische Beurteilung verzichtet werden. Hat also 
der Sponsor seinen Hauptsitz in Deutschland und wird das Forschungsprojekt in der Schweiz durchgeführt, genügt eine Beurteilung durch die zuständige EKF in der Schweiz.

Fehlt an einem Durchführungsort die gesetzliche Grundlage zur Bestimmung einer zuständigen EKF, so ist abzuklären, ob es aufgrund der Praxis der zuständigen Behörden des Gesundheitswesens und/oder der Forschung solche Kommissionen gibt, die anerkannt werden. Wurde an einem der Durchführungsorte keine zuständige EKF bestimmt oder ist keine bestimmbar (vgl. 3.3), sind in diesem Land Forschungsaktivitäten ausgeschlossen.

\subsection{Durchführungsort eines Forschungsprojektes}

Ein Forschungsvorhaben gilt an denjenigen Orten als durchgeführt, zu denen irgendeine Form von Beziehung besteht. $\mathrm{Zu}$ jeder für die Realisierung des Forschungsvorhabens notwendigen Aktivität besteht eine Beziehung. In der Praxis lassen sich zwei Arten von Beziehung unterscheiden.

Primäre Beziehung: der Ort, an dem die eigentlichen Forschungsarbeiten durchgeführt werden, d.h.

- die Rekrutierung, die Auswahl und die Betreuung von Versuchspersonen im Rahmen des Forschungsprojektes;

- die Anwendung der Testverfahren und die Erfassung von Daten und biologischen Materialien;

- die Kontrolle der Versuchspersonen nach der Studie.

Die wesentliche Funktion der EKF besteht im Schutz der Versuchspersonen. Deshalb stellt jede Aktivität, die diese Schutzfunktion tangiert, ein Kriterium der «primären Beziehung» für die Beurteilung dar.

Sekundäre Beziehung: der Ort, an dem die Forschenden oder der Sponsor normalerweise tätig sind. Dies sind zum Beispiel der Sitz des Sponsors oder das Spital beziehungsweise das Forschungszentrum, in dem der Prüfarzt seinen Beruf in der Schweiz üblicherweise ausübt.

\subsection{Bestimmung der Kommission}

Die zuständigen Ethikkommissionen werden durch die jeweilige Gesetzgebung des Landes bestimmt, in dem das Projekt beurteilt werden muss. Auch die Regeln bezüglich der Anerkennung, der Zusammensetzung und der Funktionsweise dieser Kommissionen werden durch die Gesetzgebung des jeweiligen Landes vorge- geben. Fehlen entsprechende Regeln, gilt es, sich an die Vorgehensweise der für das Gesundheitswesen und die Forschung zuständigen öffentlichen Instanzen zu halten (siehe 3.1).

\section{Umfang der Beurteilung}

\subsection{Die Art der Beziehung als Kriterium}

Um zu wissen, wie ausführlich die Beurteilung durch eine EKF auszufallen hat, muss man den Grad der Beziehung in Betracht ziehen; dieser ist für die jeweils zuständige EKF massgebend. Die Kriterien sind nicht alle gleichwertig. Das Hauptziel der ethischen Beurteilung ist der Schutz der Versuchspersonen. Elemente, die mit diesem Ziel in enger Beziehung stehen, bilden deshalb Kriterien der «primären Beziehung». Wenn die Beziehung zum Schutz der Versuchspersonen weniger eng ist, spricht man von Kriterien der «sekundären Beziehung».

Der EKF, die aufgrund einer «primären Beziehung» für ein Forschungsprojekt zuständig ist, ist am besten in der Lage, lokale, für den Schutz der Versuchspersonen relevante Faktoren zu beurteilen. Dies ist namentlich der Fall, wenn sich die Zuständigkeit der EKF aus dem Wohnsitz der Versuchspersonen, dem Durchführungsort der Forschung oder dem Ort, an dem das Forschungspersonal seine Aktivitäten ausführt, ableitet. In einer Nord-Süd-Partnerschaftsforschung wird diese Art von Beziehung vorwiegend in den südlichen Ländern zu finden sein.

Die EKF, die ihre Zuständigkeit aus einer sekundären Beziehung ableitet, ist der Zugang zu den lokalen Bedingungen, die den Versuchspersonenschutz beeinflussen können, nur schwer möglich. Folglich fehlt es ihr an der Möglichkeit, den konkreten Schutzbedarf der Versuchspersonen zu beurteilen. Dies gilt insbesondere für die Zuständigkeiten, die sich aus dem Wohn- oder Firmensitz des Sponsors, des Koordinators oder der Forschenden ableiten. In einer Nord-SüdPartnerschaftsforschung werden diese Anknüpfungsgründe vorwiegend in den nördlichen Ländern zu finden sein.

\subsection{Auf Prinzipien beschränkte Beurteilung}

Wenn sie das Studienprotokoll aufgrund einer sekundären Beziehung erhalten hat, beschränkt die EKF ihre Beurteilung auf die Grundprinzipien der Forschungsethik, wie sie in der Deklaration von Helsinki festgelegt sind. Diese Grundprinzipien gelten unabhängig von lokalen Bedingungen.

Die Deklaration von Helsinki legt Mindeststandards fest, deren Nichtbeachtung jede me- 
dizinische Forschung inakzeptabel macht. Vor diesem Hintergrund ist jede EKF verpflichtet sicherzustellen, dass diese Standards in einem konkreten Fall eingehalten werden. Eine EKF, die aufgrund einer sekundären Beziehung angefragt wurde, muss namentlich folgende Elemente prüfen: Vollständigkeit des Studienprotokolls, wissenschaftliche Validität und medizinische Relevanz des Forschungsprojektes, Nutzen-RisikoVerhältnis, mögliche Interessenkonflikte zwischen dem Sponsor, dem Koordinator und den Forschenden usw. Diese Basisprüfung darf die EKF nicht daran hindern, gewisse Elemente, die mit den lokalen Bedingungen des Durchführungsortes verknüpft sind, ebenfalls einer Prüfung zu unterziehen. Dies gilt zum Beispiel für die freiwillige und aufgeklärte Einwilligung der Versuchspersonen. Da es sich aber um Prüfung der Prinzipien handelt, ist nur zu prüfen, ob dieses Vorgehen tatsächlich auch vorgesehen ist und ob die dazu verwendeten Informationen zweckdienlich sind. Schliesslich hat sich die EKF der Tatsache zu versichern, dass das Studienprotokoll der am Durchführungsort zuständigen EKF unterbreitet wird. Zusammenfassend kann festgehalten werden, dass die EKF zu prüfen hat, ob das zu beurteilende Forschungsvorhaben auch in der Schweiz durchgeführt werden könnte, dies unter der Voraussetzung, dass die von den lokalen Bedingungen abhängigen Anforderungen berücksichtigt werden (Beispiel einer positiven Stellungnahme im Anhang).

\subsection{Vertiefte Beurteilung}

Wenn die EKF ein Studienprotokoll aufgrund einer primären Beziehung zur Beurteilung erhält, nimmt sie eine vertiefte Beurteilung vor. Tatsächlich ist die lokale EKF am besten in der Lage zu prüfen, ob die Bedingungen bzw. Anforderungen des entsprechenden Landes respektiert werden. Sie hat sich zu vergewissern, ob unter Berücksichtigung der lokalen Bedingungen - die grundlegenden Prinzipien der Forschungsethik beachtet werden. Die Prüfungspflicht betrifft Kriterien wie die Einwilligung der Versuchspersonen, die beruflichen Qualifikationen des Forschungspersonals, die Qualität der Infrastruktur oder die auf lokaler Ebene möglichen Interessenkonflikte. Ausserdem müssen der lokal zuständigen Kommission die Stellungnahmen aller anderen zuständigen EKF zugänglich sein, damit sie diese in ihre Beurteilung mit einbeziehen kann.

\section{Anhang}

\section{Beispiel einer positiven Stellungnahme bezüglich eines im Ausland durchgeführten Forschungsvorhabens}

«Das Forschungsvorhaben verstösst prinzipiell nicht gegen Anforderungen, die in der Schweiz bei Forschungsuntersuchungen mit Menschen zu beachten sind, namentlich

- wissenschaftliche Validität und Relevanz des Forschungsvorhabens und der erwarteten Resultate;

- günstiges Nutzen-Risiko-Verhältnis;

- Einwilligung der Versuchspersonen;

- Schutz der Privatsphäre und der Vertraulichkeit;

- Entschädigungsverfahren im Falle eines Schadens;

- berufliche Qualifikation der im Projekt involvierten Schweizer Forschenden;

- Definitionen der Qualifikationen, die von den anderen Forschenden gefordert werden.

Ob das Projekt unter ethischen Gesichtspunkten akzeptiert werden kann, hängt jedoch von der Berücksichtigung lokaler Bedingungen $a b$, die nicht beurteilt werden konnten. Die vorliegende Stellungnahme betrifft insbesondere die folgenden Punkte nicht:

- Relevanz des Projektes im Hinblick auf die Bedürfnisse des lokalen Gesundheitswesens;

- Verfahren zur Rekrutierung der Versuchspersonen, insbesondere die in lokaler Sprache abgefassten Informations- und Einwilligungsformulare;

- angemessene Schadensdeckung für mögliche Personenschäden (Art der gedeckten Schäden und vorgesehene Höhe des Schadenersatzes);

- die Angemessenheit der lokalen Infrastruktur (Material, Räumlichkeiten, Personal usw.) im Hinblick auf den bestmöglichen Schutz der Versuchspersonen;

- berufliche Qualifikation des nichtschweizerischen Forschungspersonals.

Sämtliche aufgelisteten Punkte bedürfen einer vertieften Beurteilung durch die am (an den) Durchführungsort(en) zuständige(n) EKF.»

Diese Präzisierungen können direkt in das Fenster «Auflagen» der Entscheidmitteilung eingefügt werden. 